\title{
Safety and efficacy analysis of microwave ablation in small hepatocellular carcinomas sized below $3 \mathrm{~cm}$
}

\author{
Ferhat C. Piskin ${ }^{A, B, C, D, E, F, G}$, Huseyin T. Balli ${ }^{A, B, D, E, G}$, Kairgeldy Aikimbaev ${ }^{A, C, D, E}$ \\ Department of Radiology, Cukurova University Medical Faculty, Turkey
}

\section{Abstract}

Purpose: The aim of this study was to investigate the efficacy and safety of microwave ablation (MWA) in small hepatocellular carcinomas sized $\leq 3 \mathrm{~cm}$, determine long-term survival, and identify prognostic factors for survival rates.

Material and methods: In this study, the radiological and laboratory findings obtained from 31 consecutive patients who underwent MWA were retrospectively evaluated. The survival periods and complication rates were analysed.

Results: Microwave ablation was applied to 42 hepatocellular carcinoma nodules in 31 patients. The mean age of the patients was $61 \pm 7.3$ (median 62, range 46-78) years. The mean overall survival (OS) was $47.4 \pm 3.3$ months. The rates of cumulative OS in the first, second, and third years were $95.2 \%, 91.8 \%$, and $79.2 \%$, respectively. The mean disease-free survival (DFS) rate was $24.1 \pm 2.5$ months. The cumulative DFS rates in the first, second, and third years were $75.6 \%, 52.5 \%$, and $28.2 \%$, respectively. The number of tumours and tumour distribution were determined as prognostic factors. No major complication was detected, but six patients (13.9\%) developed minor complications after MWA.

Conclusions: Microwave ablation in patients with hepatocellular carcinoma is a safety treatment modality with very low rates of complications. It offers an effective treatment with a high rate of complete response and local disease control according to the short-term results. In the long term, it prolongs the survival time of the treated patients. The number of tumours and tumour distribution were determined as prognostic factors affecting survival rates.

Key words: microwave ablation, hepatocellular carcinomas, long-term survival, prognostic factors.

\section{Introduction}

Hepatocellular carcinoma (HCC) is the most common primary malignant tumour of the liver, causing more than 800,000 deaths every year [1]. In the past, HCC was known to be a fatal disease with a poor prognosis because it was mostly detected in advanced stages. However, this situation has changed in recent years through screening and monitoring programs. Today, $30-60 \%$ of HCCs detected in developed countries are at an early stage. Surgical treatment methods and ablation therapy can provide cure in patients with early stage HCCs, and a survival time reaching more than five years can be achieved [2-4].

In early stage HCCs, ablation methods have come to the forefront and started to be frequently used due to their significant advantages, including their less invasive and reproducible nature compared to surgical treatment methods. Radiofrequency ablation (RFA) is a relatively older method of ablation and has been used for many years in patients with early stage HCCs [5].

Microwave ablation (MWA) is a relatively new method of ablation. It provides more homogenous, higher temperatures within the tumour in a shorter time compared to RFA. In addition, it theoretically offers more effective ablation in tumours adjacent to large vessels because it is less affected by the heat shrink effect [6]. There are studies in the literature suggesting that MWA can be used as an effective and safe treatment modality in patients with HCCs, but studies reporting the results of MWA treatment in small HCCs $(\leq 3 \mathrm{~cm})$ are limited [2-7].

\section{Correspondence address:}

Dr Ferhat Can Piskin, Department of Radiology, Cukurova University Medical Faculty, Turkey, e-mail: ferhatcpiskin@gmail.com

Authors' contribution:

A Study design · B Data collection · C Statistical analysis · D Data interpretation - E Manuscript preparation · F Literature search · G Funds collection 
The aim of this study was to investigate the efficacy and safety of MWA procedures for small HCCs $(\leq 3 \mathrm{~cm})$, determine the long-term survival, and identify prognostic factors for patients' suvival after the MWA treatment.

\section{Material and methods}

This study was planned as a single-centre, retrospective study and conducted in accordance with the principles of the Helsinki Declaration after obtaining the approval of the local clinical research Ethics Committee (Number: 0210201882/14). In this study, 31 consecutive patients who underwent the MWA procedure in the interventional radiology unit between March 2013 and November 2018 were evaluated.

\section{Patient population}

The diagnosis was made according to the guidelines of the European Association for the Study of the Liver [8]. Patients with a tumour size of $3 \mathrm{~cm}$ or less and preserved liver function (Child-Pugh class A or B), for whom surgical treatment was not indicated because of comorbidity status, were considered to be appropriate for MWA treatment and included in the study. Patients with refractory ascites, those with a bleeding disorder (indicated by an international normalised ratio of $>1.5$ and/or platelet value below $50,000 / \mathrm{mm}^{3}$ ), and those with tumour thrombus, who were not considered appropriate for MWA treatment, were excluded from the study.

\section{Microwave ablation procedure}

Informed consent was obtained from all patients before treatment. The patients were evaluated using ultrasound (US) for tumour location and size, adjacent structures, appropriate site for antenna entry, and tract. All ablation procedures were percutaneously performed using a 3.5-5 MHz convex probe under the guidance of a US device (GE Logiq 7, GE Healthcare, WI) after inducing sedoanalgesia in patients (intravenous propofol [Corden Pharma SPA, Italy] and fentanyl [Yichang Renfu Pharmaceuticals, Huibei, China]).

For the MWA procedure, the Emprint MWA system (Covidien Boulder, CO) capable of generating $100 \mathrm{~W}$ energy at $2450 \mathrm{MHz}$ or the AveCure MWA system (Medwaves, CA) capable of generating $75 \mathrm{~W}$ energy at $915 \mathrm{MHz}$ was used. As microwave antennas, $14 \mathrm{G}$ internally cooled trocar-type percutaneous antennas were used. The ablation zone was determined to cover the entire tumour and $1 \mathrm{~cm}$ of the non-tumoral parenchyma as much as possible. When the appropriate ablation zone was obtained, the tract ablation was performed before the removal of the antenna to prevent tumour seeding and bleeding due to major vessel damage.

After MWA, the patients were kept under observation for 24 hours, and those with severe pain were adminis- tered an intravenous injection of $100 \mathrm{mg}$ Contramal, and those with mild pain were given intravenous $50 \mathrm{mg} / 2 \mathrm{ml}$ dexketoprofen for analgesia. In addition, a single prophylactic dose of $500 \mathrm{mg}$ cefazolin was administered intramuscularly. The next day, the patients were evaluated using physical examination and US, and after confirming that they did not have any major complication they were discharged from the hospital.

\section{Follow-up and evaluation}

The patients were followed-up by laboratory tests and MRI at the first month after treatment, and then every third consecutive month. Liver functions and tumour markers were checked in laboratory tests. Contrast dynamic imaging was performed with a 1.5-Tesla Signa (GE Medical Systems, WI) or 3-Tesla Philips Achieva scanner (Phillips Medical Systems, The Netherlands) to evaluate treatment efficacy and recurrence.

Treatment efficacy was determined using the terminology recommended in the Tumour Ablation Manual [9]. At the first-month follow-up, the absence of contrast enhancement within the tumour was evaluated as a complete response, and partial enhancement as a partial response. The ablation procedure was repeated in patients with a partial response. After observing a complete response, the detection of contrast enhancement in the parenchyma in the ablation zone or its vicinity during the follow-up was accepted as local recurrence. Newly developed tumours in the liver parenchyma but far from the ablation zone were considered intra-hepatic recurrence.

Complications were classified according to the guidelines of the Society of Interventional Radiology Clinical Practice Guidelines [10].

\section{Statistical analysis}

In the descriptive statistics, continuous variables were expressed as mean and standard deviation values, and categorical variables as numbers and percentages. The survival time was analysed by the Kaplan-Meier method, and the effects of categorical variables on survival were evaluated using the log-rank test. The $\chi^{2}$ and Fisher's exact $\chi^{2}$ tests were used to determine whether there was a statistical difference between categorical variables. In all statistical tests, $p$ values less than 0.05 were considered statistically significant. For the statistical analysis of the data, TURCOSA software (Turcosa Analytical Ltd., Turkey) was used.

\section{Results}

In this study, the MWA treatment response and complication rates of 31 patients with 42 HCC nodules were evaluated. The demographic and clinical characteristics of the patients are summarised in Table 1. Additionally, Eastern Cooperative Oncology Group performance scale 
score ranged from 0 to 2 for all patients. Before treatment, mild ascites (3.2\%), severe hypoalbuminaemia $(<2.8 \mathrm{~g} / \mathrm{dl})(3.2 \%)$, and severe bilirubin elevation $(>3 \mathrm{~g} / \mathrm{dl})$ $(3.2 \%)$ were detected in one patient each. Table 2 summarises the characteristics of the treated tumours at the time of diagnosis. Briefly, the mean size of MWA-treated tumours was $1.92 \pm 0.4 \mathrm{~cm}$ (median 1.9, range $1.3-3.0 \mathrm{~cm}$ ).

In this study, technical success was achieved in all MWA-treated cases, and the mean ablation time was calculated as $5.6 \pm 2.6 \mathrm{~min}$ (median 5 , range 2-15 min). Mortality and major complications did not occur after MWA. Six patients (14.3\%) developed minor complications after MWA: pleural effusion (4/6), biliary dilatation (1/6), and subcapsular haematoma (1/6). All minor complications were spontaneously resolved during the follow-up without treatment.

The mean follow-up period after MWA was $31.5 \pm$ 14.4 months (median 29, range 8-66 months). During the follow-up, 11 (35.4\%) patients died, and one patient (3.2\%) was excluded from further analysis due to undergoing liver transplantation. In the first-month radiological evaluation of MWA-treated tumours, 41 tumours (97.6\%) had a complete response and one $(2.4 \%)$ had a partial response (Figures 1 A-D). During the follow-up, local recurrence occurred in three tumours (7.1\%) and intra-hepatic recurrence was observed in 21 (50\%). In addition, colon metastasis was detected in one case (2.4\%) and lung metastasis in two cases (4.8\%).

When the Kaplan-Meier survival analysis was performed, the mean overall survival (OS) time was calculated as $47.4 \pm 3.3$ months (Figure 2 ). The cumulative OS rates in the first, second, and third years was $95.2 \%$, $91.8 \%$, and $79.2 \%$, respectively. When the effects of clinical and tumour characteristics on survival were examined, the number of tumours and tumour distribution in the liver were determined as prognostic factors. The OS time was significantly higher in patients with a single tumour compared to those with $2-3$ tumours ( $50.3 \pm 1.8$ vs. 39.4 \pm 3.8 months, respectively; $p=0.018$ ), and it was also significantly higher in patients with bilobar distribution compared to those with unilobar tumours $(48.9 \pm 2.2$ vs. $39 \pm 4.3$ months, $p=0.029$ ) (Figures 3 and 4 ). The baseline $\alpha$-fetoprotein (AFP) value, Child-Pugh score, and hepatitis aetiology did not have a significant effect on the survival time of patients who underwent MWA $(p=0.614$, $p=0.999$, and $p=0.669$, respectively) (Table 3 ).

The Kaplan-Meier survival analysis revealed the mean disease-free survival (DFS) as $24.1 \pm 2.5$ months (Figure 5). The cumulative DFS rates in the first, second, and third years were $75.6 \%, 52.5 \%$, and $28.2 \%$, respectively. The patients with a single tumour had significantly higher DFS time than those with $2-3$ tumours $(33.2 \pm 2.6$ vs. $16.5 \pm 2.4$ months, respectively; $p=0.0002$ ), and the patients with unilobar tumour distribution had a significantly higher DFS time than those with bilobar tumours $(27.2 \pm 2.7$ vs. $17.4 \pm$ 3.2 months, respectively; $p=0.029$ ) (Figures 6 and 7).
Table 1. Demographic and clinical characteristics of the patients

\begin{tabular}{|l|c|}
\hline Variable & Results \\
\hline Age (years), mean \pm SD & $61 \pm 7.3$ \\
\hline \begin{tabular}{l} 
Gender, $n$ (\%) \\
\hline Male
\end{tabular} & $26(83.9)$ \\
\hline Female & $5(16.1)$ \\
\hline \begin{tabular}{l} 
Child-Pugh, $n$ (\%) \\
\hline A
\end{tabular} \\
\hline B & $26(83.9)$ \\
\hline Hepatitis aetiology, $n(\%)$ & $5(16.1)$ \\
\hline Hepatitis B virus & $24(77.4)$ \\
\hline Hepatitis C virus & $7(22.6)$ \\
\hline Alpha-fetoprotein (ng/ml), $n(\%)$ & $23(74.2)$ \\
\hline$\leq 20$ & $8(25.8)$ \\
\hline$>20$ &
\end{tabular}

$S D$ - standard deviation.

Table 2. Characteristics of microwave ablation-treated tumours

\begin{tabular}{|l|l|}
\hline $\begin{array}{l}\text { Variable } \\
\text { Number of tumours, } n(\%)\end{array}$ & $17(40.5)$ \\
\hline 1 & $25(59.5)$ \\
\hline $2-3$ & \multicolumn{2}{|l|}{} \\
\hline Tumour distribution, $n(\%)$ & $25(59.5)$ \\
\hline Unilobar & $17(40.5)$ \\
\hline Bilobar & $1.9 \pm 0.4$ \\
\hline Tumour size $(\mathrm{cm})$, mean \pm SD & \\
\hline
\end{tabular}

SD - standard deviation.

\section{Discussion}

Hepatocellular carcinoma is the most common primary malignant tumour of the liver and the fifth most common cancer. Ablation treatment has become one of the first treatment options for tumours of $\leq 3 \mathrm{~cm}$ in size because this method is less invasive and more reproducible compared to surgical procedures [11].

The incidence of HCC was found to be two to three times higher in the male population than in females [12]. Similarly, in our study, the male patients constituted the majority $(83.9 \%)$, and the male/female ratio was even higher than reported in the literature. This is because the male/female ratio of viral hepatitis is higher in Turkey compared to other countries [13]. Because HBV infection is endemic in Turkey, the incidence of HBV was higher in our sample with HCCs compared to the literature; the causative agent was detected as $\mathrm{HBV}$ in 24 patients (77.4\%) and HCV in seven (22.6\%) [14].

With the development of MWA technology used in HCC patients over the years, positive results have been 

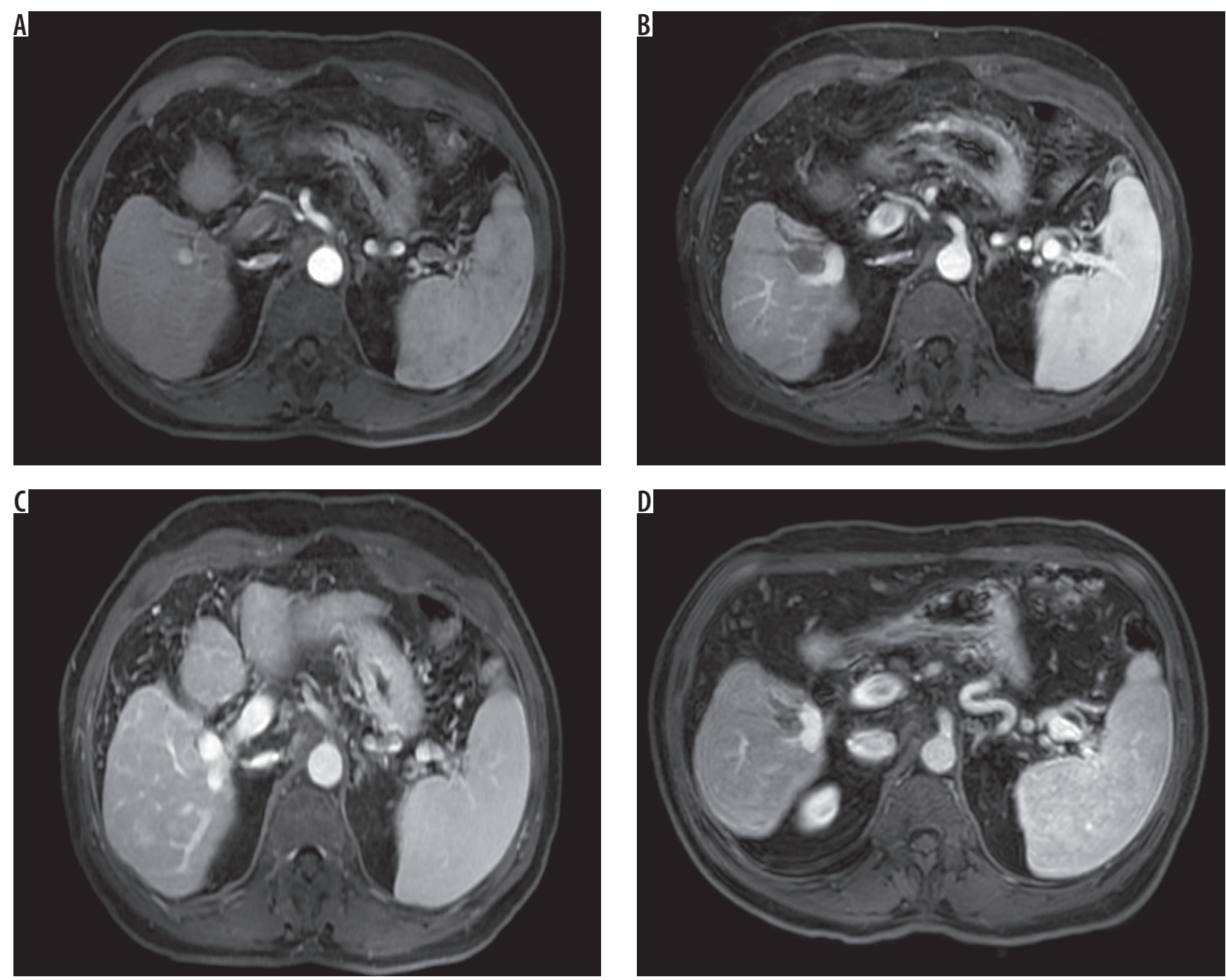

Figure 1. A 61-year-old male patient was treated with microwave ablation after the detection of hepatocellular carcinoma in magnetic resonance imaging. A) On axial T1-weighted early arterial phase image, a hyperintense nodule lesion (diameter: $1.5 \mathrm{~mm}$ ) was detected in segment five. B) 0n axial T1-weighted venous phase, the lesion was washed out of contrast material. C, D) On the axial T1-weighted portal phase image, the lesion was complete ablate at the first and $24^{\text {th }}$ month after treatment

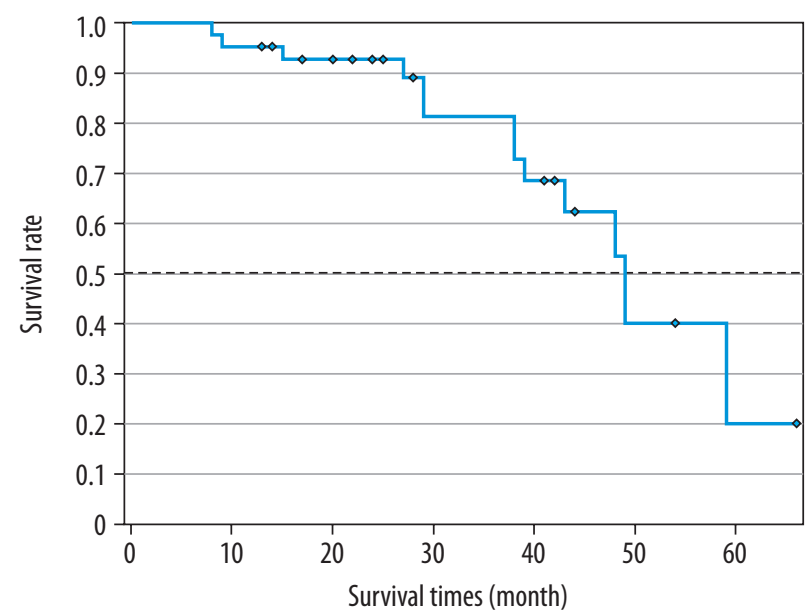

Figure 2. Overall survival times of microwave ablation-treated hepatocellular carcinoma patients

increasingly obtained in terms of local disease control and early outcome. First, in their study in 2002, Shibata et al. applied MWA to 46 HCC nodules of $\leq 3 \mathrm{~cm}$ and found a complete response in 41 (89.1\%) [15]. More recently, in a 2014 study, Abdelaziz et al. achieved a complete response in $54(98.2 \%)$ of 55 HCC nodules of $\leq 3 \mathrm{~cm}$ after MWA treatment [16]. Internally cooled antenna systems used in MWA systems have enabled the ablation energy to be distributed more homogenously within the tumour compared to old antenna systems, thus resulting in higher efficacy in the outer parts of the tumour. Therefore, the rates of complete response rates have increased over the years. However, as tumour size increases $(>3 \mathrm{~cm})$, the complete response rate and the early efficacy of MWA in local disease control is reduced due to insufficient energy distribution within the tumour. Medhat et al. applied MWA to 26 HCCs between 5 and $7 \mathrm{~cm}$ and achieved a complete response in $19(73.1 \%)$ tumours [17]. Liu et al. calculated the complete response rates as $75 \%(21 / 28)$ for $5-8 \mathrm{~cm}$ tumours and $94.2 \%$ (45/48) for 3-5 cm tumours. A significant difference was detected in the complete response rates 
Table 3. Effect of clinical and tumour characteristics on survival time

\begin{tabular}{|c|c|c|c|c|}
\hline Variable (mean \pm SD) & Overall survival time (months) & $p$-value & Disease-free survival time (months) & $p$-value \\
\hline \multicolumn{5}{|l|}{ Number of tumours } \\
\hline 1 & $50.3 \pm 1.8$ & 0.018 & $33.2 \pm 2.6$ & \multirow[t]{2}{*}{0.0002} \\
\hline $2-3$ & $39.4 \pm 3.8$ & & $16.5 \pm 2.4$ & \\
\hline \multicolumn{5}{|l|}{ Tumour distribution } \\
\hline Unilobar & $48.9 \pm 2.2$ & 0.029 & $27.2 \pm 2.7$ & \multirow[t]{2}{*}{0.029} \\
\hline Bilobar & $39 \pm 4.3$ & & $17.4 \pm 3.2$ & \\
\hline \multicolumn{5}{|l|}{$\alpha$-fetoprotein (ng/ml) } \\
\hline$\leq 20$ & $47.1 \pm 3.1$ & 0.614 & $23.3 \pm 2.6$ & 0.794 \\
\hline$>20$ & $43.2 \pm 6.4$ & & $24 \pm 4.3$ & \\
\hline \multicolumn{5}{|l|}{ Hepatitis aetiology } \\
\hline Hepatitis B virus & $46.6 \pm 3.1$ & 0.999 & $24 \pm 2.5$ & 0.459 \\
\hline Hepatitis C virus & $47.2 \pm 6.3$ & & $21.1 \pm 4.5$ & \\
\hline \multicolumn{5}{|l|}{ Child-Pugh } \\
\hline $\mathrm{A}$ & $47.2 \pm 3.5$ & 0.669 & $23 \pm 2.3$ & 0.807 \\
\hline$B$ & $44 \pm 7.5$ & & $24 \pm 6.5$ & \\
\hline
\end{tabular}

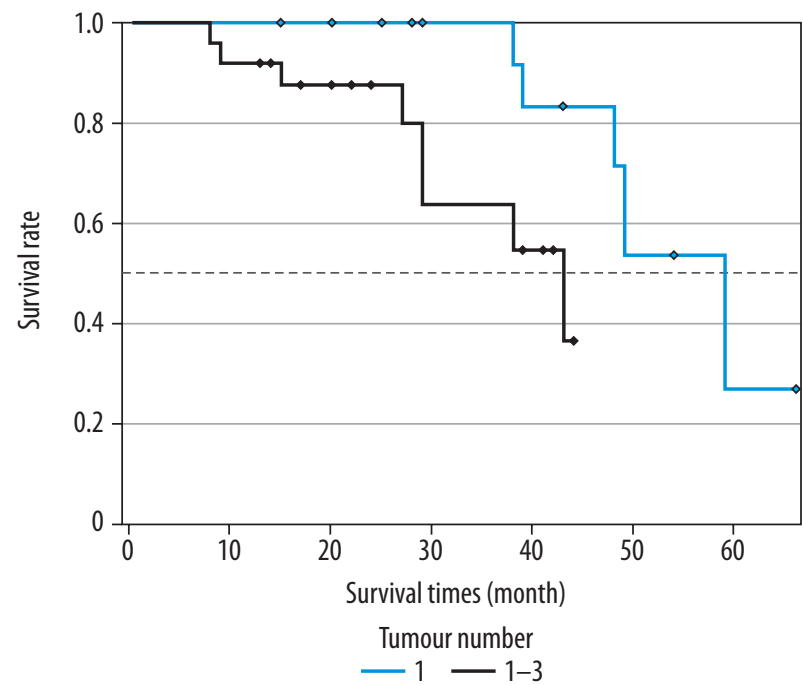

Figure 3. Overall survival times of microwave ablation-treated hepatocellular carcinoma patients according to the number of tumours

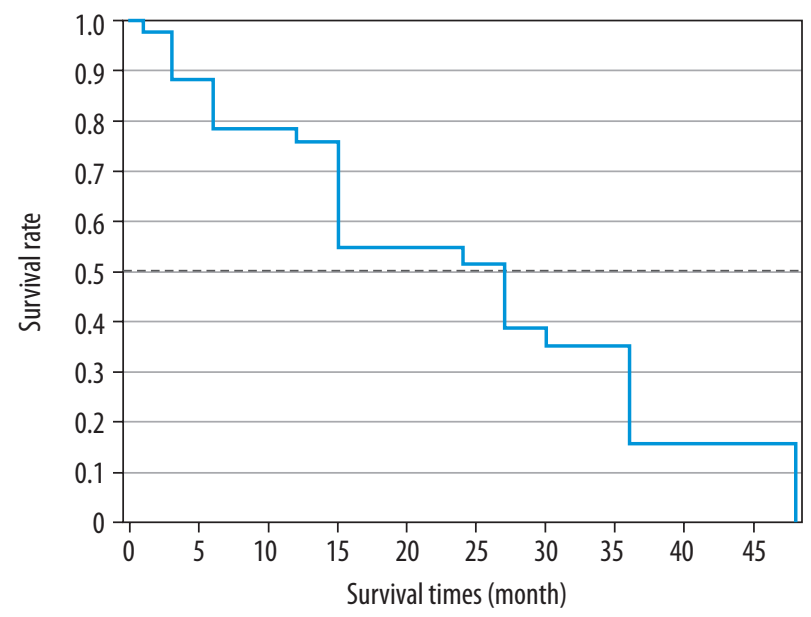

Figure 5. Disease-free survival times of microwave ablation-treated hepatocellular carcinoma patients

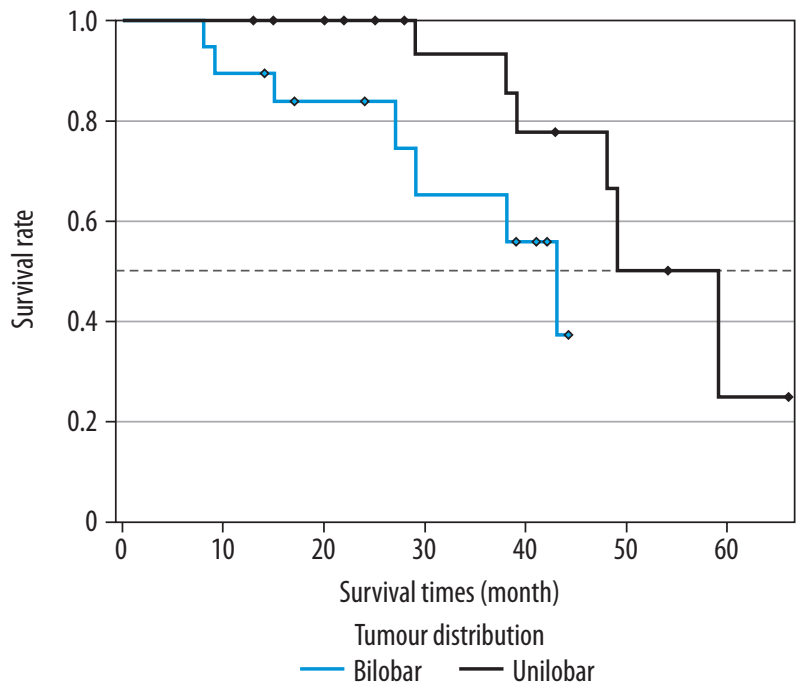

Figure 4. Overall survival times of microwave ablation-treated hepatocellular carcinoma patients according to tumour distribution

of these two groups with a different tumour size range $(p=0.033)$ [18]. All of the MWA antennas used in our study were internally cooled, and the mean size of HCC nodules was $1.9 \pm 0.4 \mathrm{~cm}$ (median 1.9 , range $1.3-3 \mathrm{~cm}$ ). In the evaluation of early results, 41 (97.6\%) of the tumours had a complete response, and one (2.4\%) had a partial response. Considering the data in the literature and the early results of our study, MWA presents as a very effective treatment modality in terms of local disease control in HCCs of $\leq 3 \mathrm{~cm}$. However, as the tumour size increases, the early success rate (complete response rate) decreases.

While the early results of MWA are positive in patients with HCCs, recurrences reaching $50 \%$ are detected in the long-term follow-up. Sun et al., who evaluated 182 patients who underwent MWA, achieved a 93\% complete 


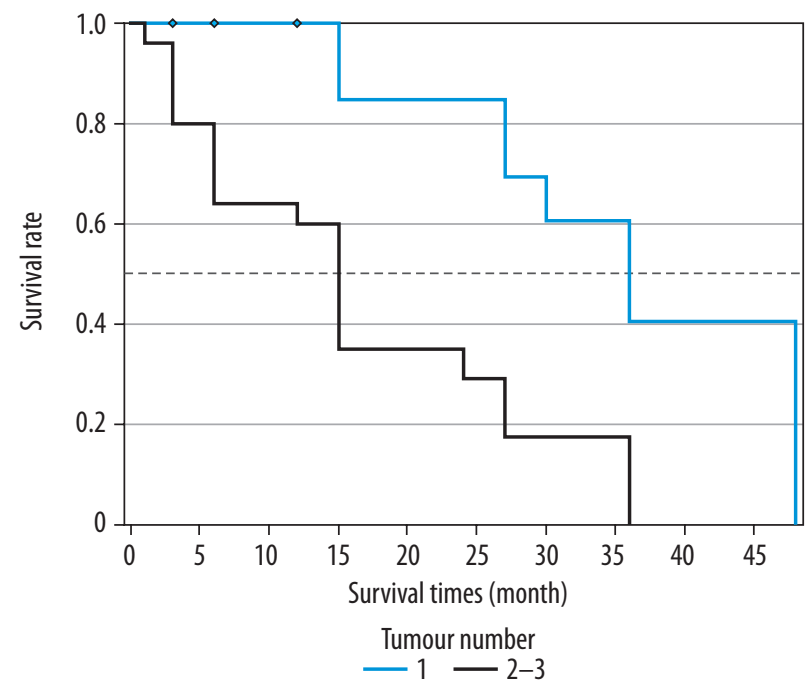

Figure 6. Disease-free survival times of microwave ablation-treated hepatocellular carcinoma patients according to the number of tumours

response rate in the first-month evaluation, but during the later follow-up, $54.2 \%$ of the patients developed intrahepatic recurrence [19]. In a study by Abdelaziz et al., local tumour recurrence was found as $3.9 \%$ and intrahepatic recurrence as $13.6 \%$ [16]. In MWA-treated HCCs, Ding et al. detected the OS rates as $98 \%, 90.7 \%$, and $77.6 \%$ for the first, second, and third years, respectively, and the DFS rates were $75 \%, 59.4 \%$, and $32.1 \%$, respectively. In addition, the mean OS time was $45.3 \pm 2.2$ months, and the mean DFS time was $25 \pm 1.6$ months [20]. In the current study, local tumour recurrence was detected in three tumours $(7.1 \%)$ and intra-hepatic recurrence in $21(50 \%)$, and the OS and DFS times were calculated as $47.4 \pm 3.3$ months and $24.1 \pm 2.5$ months, respectively, which is consistent with the long-term results of previous studies in the literature. The success rate of HCC treatment in terms of OS time is low due to recurrence in the long term because these nodules frequently develop on the background of cirrhotic liver parenchyma. However, because MWA can be repeated multiple times even in patients with decreased liver parenchymal reserve, longer OS times can be obtained in early-stage tumours.

Since the group of HCC patients is heterogeneous and the disease has different treatment options with varying efficacy, studies have been conducted to identify prognostic factors for appropriate treatment and patient selection and to examine their effects on survival. Xu et al. applied thermal ablation treatment (RFA and MWA) to $137 \mathrm{pa}-$ tients and evaluated 16 possible prognostic factors at the end of the four-year follow-up [21]. The serum AFP value and Child-Pugh score at the time of diagnosis were reported to be predictive factors of prognosis. In an article published in 2017, Ma et al. treated 433 patients using MWA, and as a result, they identified the prognostic factors as the number of tumours, a tumour size of $>5 \mathrm{~cm}$, and a high AFP value at the time of diagnosis [22]. In the present study, the number of tumours and tumour distri-

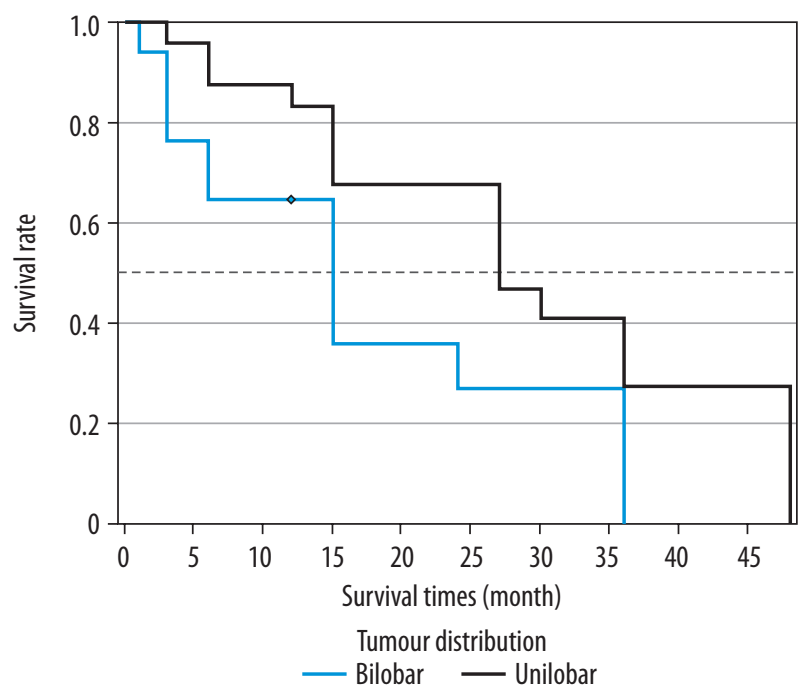

Figure 7. Disease-free survival times of according to tumour distribution

bution, aetiology of hepatitis, AFP values, and Child-Pugh score at diagnosis were evaluated as possible prognostic factors. The number of tumours and tumour distribution at the time of diagnosis were determined as prognostic factors affecting OS and DFS times. A tumour count of more than 1 and bilobar distribution of HCC nodules in the liver is a common disease marker. The risk of recurrence is higher in these patients. In this study, the AFP values and Child-Pugh score did not present as prognostic factors. In the literature, it was found that in patients with HCCs, the serum AFP values were increased in the active disease state (necrosis and regeneration). Therefore, serum AFP values are important markers in terms of disease prognosis at the time of diagnosis and recurrence in follow-up [21]. Since the Child-Pugh score offers a clue to possible liver failure, it can be predicted that patients with high tumour-independent Child-Pugh score will have shorter survival times associated with liver failure.

In an article published to create a common reporting language in patients who underwent ablation, Ahmed et al. considered as major complications those that caused morbidity and required hospitalisation or additional treatment. The authors included the remaining complications in the category of minor complications [9]. In a systematic meta-analysis by Bertot et al., studies conducted between 1982 and 2010 were compiled and a total of 1185 MWA procedures were reviewed. The major complication rate was found as $4.6 \%$ and the mortality rate as $0.23 \%$ [23]. No vascular complications were observed in our study. This was achieved because the pre-procedural bleeding profiles of all patients were within the normal range and the needle tract was cauterised by ablation at the end of the procedure.

Minor complications are those that do not require additional treatment, such as subcapsular hematoma, perihepatic collection, skin burns, and ipsilateral pleural effusion [9]. These complications do not cause additional 
morbidity as long as they are controlled. In this study, minor complications (pleural effusion, perihepatic collection, and biliary dilatation) developed in six patients (14.2\%) after MWA treatment.

Post-treatment fever and abdominal pain indicate postablation syndrome and are defined as side effects. These side effects can be controlled by keeping the patient under observation for the first 24 hours and administering low-dose anti-inflammatory medication [9]. In this study, prophylactic analgesia and anti-inflammatory treatment were applied to all patients; thus, possible side effects were prevented.

There were some limitations to our study. Due to the retrospective nature of the study, the appropriate sample size was not calculated before the study. In addition, the 5- and 10-year long-term results of patients who underwent MWA were not available. Therefore, there was only limited information about the local recurrence of the patients in some periods.

\section{Conclusion}

MWA is a safe treatment modality in patients with HCCs because it has a very low risk of complications, and in the event of complications, appropriate and timely treatment completely restores the patients' health. MWA is also an effective treatment modality in patients with HCCs considering that the short-term results indicate a high rate of complete response and local disease control, while the long-term results reveal prolonged survival time in MWA-treated patients. The number of tumours and tumour distribution were determined as prognostic factors affecting survival rates.

\section{Conflict of interest}

The authors report no conflict of interest.

\section{References}

1. Bray F, Ferlay J, Soerjomataram I, et al. Global cancer statistics 2018 GLOBOCAN estimates of incidence and mortality worldwide for 36 cancers in 185 countries. CA Cancer J Clin 2018; 68: 394-424.

2. Bruix J, Sherman M. Management of hepatocellular carcinoma: an update. Hepatology 2011; 53: 1020-1022.

3. Farinati F, Sergio A, Baldan A, et al. Early and very early hepatocellular carcinoma: when and how much do staging and choice of treatment really matter? A multi-center study. BMC Cancer 2009; 9: 33.

4. Xu Y, Shen Q, Wang N, et al. Microwave ablation is as effective as radiofrequency ablation for very-early-stage hepatocellular carcinoma. Chin J Cancer 2017; 36: 14.

5. Lei JY, Wang WT, Yan LN, et al. Radiofrequency ablation versus surgical resection for small unifocal hepatocellular carcinomas. Medicine (Baltimore) 2014; 93: e271.

6. Lubner MG, Brace CL, Hinshaw JL, et al. Microwave tumor ablation: mechanism of action, clinical results, and devices. J Vasc Interv Radiol 2010; 21 (Suppl 8): S192-203.

7. Fan W, Li X, Zhang L, et al. Comparison of microwave ablation and multipolar radiofrequency ablation in vivo using two internally cooled probes. AJR Am J Roentgenol 2012; 198: 46-50.

8. European Association for the Study of the Liver, European Organisation for Research and Treatment of Cancer. EASL-EORTC clinical practice guidelines: management of hepatocellular carcinoma. J Hepatol 2012; 56: 908-943.

9. Ahmed M, Solbiati L, Brace CL, et al. Image-guided tumor ablation: standardization of terminology and reporting criteria - a 10-year update. Radiology 2014; 273: 241-260.

10. Cardella JF, Kundu S, Miller DL, et al. Society of interventional radiology clinical practice guidelines. J Vasc Interv Radiol 2009; 20: 189-191.

11. Bruix J, Sherman M. Management of hepatocellular carcinoma: an update. Hepatology 2011; 53: 1020-1022.

12. Ghouri YA, Mian I, Rowe JH. Review of hepatocellular carcinoma: epidemiology, etiology, and carcinogenesis. J Carcinog 2017; 16: 1.
13. Alacacioglu A, Somali I, Simsek I, et al. Epidemiology and survival of hepatocellular carcinoma in Turkey: outcome of multicenter study. Jpn J Clin Oncol 2008; 38: 683-688.

14. Akkiz H, Carr BI, Yalcin KK, et al. Characteristics of hepatocellular carcinoma aggressiveness factors in turkish patients. Oncology 2018; 94: 116-124.

15. Shibata T, Iimuro Y, Yamamoto Y, et al. Small hepatocellular carcinoma: comparison of radio-frequency ablation and percutaneous microwave coagulation therapy. Radiology 2002; 223: 331-337.

16. Abdelaziz A, Elbaz T, Shousha HI, et al. Efficacy and survival analysis of percutaneous radiofrequency versus microwave ablation for hepatocellular carcinoma: an Egyptian multidisciplinary clinic experience. Surg Endosc 2014; 28: 3429-3434.

17. Medhat E, Abdel Aziz A, Nabeel M, et al. Value of microwave ablation in treatment of large lesions of hepatocellular carcinoma. J Dig Dis 2015 ; 16 : 456-463.

18. Liu Y, Zheng Y, Li S, et al. Percutaneous microwave ablation of larger hepatocellular carcinoma. Clin Radiol 2013; 68: 21-26.

19. Sun AX, Cheng ZL, Wu PP, et al. Clinical outcome of medium-sized hepatocellular carcinoma treated with microwave ablation. World J Gastroenterol 2015; 21: 2997-3004.

20. Ding J, Jing X, Liu J, et al. Comparison of two different thermal techniques for the treatment of hepatocellular carcinoma. Eur J Radiol 2013; 82: 1379-1384.

21. Xu HX, Lu MD, Xie XY, et al. Prognostic factors for long-term outcome after percutaneous thermal ablation for hepatocellular carcinoma: a survival analysis of 137 consecutive patients. Clin Radiol 2005; 60: 1018-1025

22. Ma S, Ding M, Li J, et al. Ultrasound-guided percutaneous microwave ablation for hepatocellular carcinoma: clinical outcomes and prognostic factors. J Cancer Res Clin Oncol 2017; 143: 131-142.

23. Bertot LC, Sato M, Tateishi R, et al. Mortality and complication rates of percutaneous ablative techniques for the treatment of liver tumors: a systematic review. Eur Radiol 2011; 21: 2584-2596. 